\title{
Gravitational Space-Time Curve Generation via Accelerated Charged Particles
}

\author{
Edward A. Walker \\ Mathematics Department, Florida Memorial University, Miami Gardens, USA \\ Email: dwrdwalker@yahoo.com
}

Received 8 April 2016; accepted 10 May 2016; published 13 May 2016

Copyright (C) 2016 by author and Scientific Research Publishing Inc.

This work is licensed under the Creative Commons Attribution International License (CC BY). http://creativecommons.org/licenses/by/4.0/

(c) (i) 0 pen Access

\section{Abstract}

A force with an acceleration that is equal to multiples greater than the speed of light per unit time is exerted on a cloud of charged particles. The particles are resultantly accelerated to within an infinitesimal fraction of the speed of light. As the force or acceleration increases, the particles' velocity asymptotically approaches but never achieves the speed of light obeying relativity. The asymptotic increase in the particles' velocity toward the speed of light as acceleration increasingly surpasses the speed of light per unit time does not compensate for the momentum value produced on the particles at sub-light velocities. Hence, the particles' inertial mass value must increase as acceleration increases. This increase in the particles' inertial mass as the particles are accelerated produce a gravitational field which is believed to occur in the oscillation of quarks achieving velocities close to the speed of light. The increased inertial mass of the density of accelerated charged particles becomes the source mass (or Big "M") in Newton's equation for gravitational force. This implies that a space-time curve is generated by the accelerated particles. Thus, it is shown that the acceleration number (or multiple of the speed of light greater than 1 per unit of time) and the number of charged particles in the cloud density are surjectively mapped to points on a differential manifold or space-time curved surface. Two aspects of Einstein's field equations are used to describe the correspondence between the gravitational field produced by the accelerated particles and the resultant space-time curve. The two aspects are the Schwarzchild metric and the stress energy tensor. Lastly, the possibility of producing a sufficient acceleration or electromagnetic force on the charged particles to produce a gravitational field is shown through the Lorentz force equation. Moreover, it is shown that a sufficient voltage can be generated to produce an acceleration/force on the particles that is multiples greater than the speed of light per unit time thereby generating gravity.

\section{Keywords}

Charged Particles, Accelerated Particles, Inertial Mass, Gravitational Force, Einstein's Field Equations, Space-Time Manifold, Schwardchild Metric, Stress Energy Tensor, Surjective Mapping, 


\section{Lorentz Force}

\section{Introduction}

It has been shown that a gravitational field can be generated by the oscillation of a quark in a paper written by author Eli Peter Manor published in 2016 in the Journal of Modern physics [1]. While oscillating, the quark would achieve velocities that near the speed of light; the inertial mass of the particle would increase resultantly generating a gravitational field [1]. The aim of this paper is to show that a gravitational field can also be produced as the inertial mass of a charged particle increases when accelerated to the verge of the speed of light via an electromagnetic field (as in a particle accelerators). Moreover, a description of the space-time curve associated with the gravitational field generated will be mathematically formulated.

In describing the assertion of this paper in more detail; a gravitational field is generated when a cloud of charged particles is accelerated to the precipice of the speed of light. The acceleration enacted on the particles exceed the speed of light per unit time, however massive particles cannot exceed the speed of light as is well known. Resultantly, as the acceleration increasingly exceeds the speed of light per unit time, the particles' velocities approaches but never achieves a luminous velocity. Mathematically, the particles' velocities asymptotically approaching the speed of light will not compensate for the amount of force or acceleration exerted on the particles; the inertial mass value of each particle must increase to compensate for the increasing acceleration or force. In this assertion, each charged particles' velocity is approximated to a constant $99 \%$ of the speed of light $(v \approx 0.99 c)$, allowing the measurement of increased inertial particle mass. Consider Newtonian gravitational force as shown below [2].

$$
F_{g}=\frac{G M m}{r^{2}}
$$

All mass values correspond to a density value; even if the unit volume is infinitesimally small. The sum total of increasing individual inertial mass values of each particle in the density of accelerated particles is set equal to source mass $M$ in the gravitational force $F_{g}$ as the acceleration on the charged particles increase. Hence, as the force acting on the particles increase with an invariant approximate velocity of $99 \%$ the speed of light; the corresponding force of gravity increases proportionally. This implies that a curvature in space-time is also generated by the cloud of accelerated charged particles. Therefore, Einstein's field equations are used to describe the curvature in space-time generated by the accelerated charged particles. It will be shown that there exist a surjective or "onto" map from the Cartesian product of the particles acceleration number (or the multiples of acceleration past the speed of light per unit time) and the number of particles in the cloud density to the codomain of points and displacements on a space-time manifold. Two descriptions using Einstein's field equation are shown to correlate to the surjective mapping from the domain of the particle acceleration number and the number of particles to the codomain of points or displacements on the space-time curve produced by the accelerated cloud. The first description is the Schwarzchild metric description and the second is the stress-energy tensor description. Lastly, the electromagnetic force or Lorentz force equation is used to show that it is possible to obtain a sufficient voltage to accelerate a cloud of charged particles to a velocity that is an infinitesimal fraction below the speed of light to produce a gravitational field and/or space-time curve.

\section{Acceleration of Charged Particles and Newtonian Gravitation}

In using electromagnetic force or Lorentz force to accelerate the cloud of charged particles, acceleration $a_{x}$ is generated. Acceleration $a_{x}$ is $N_{a}$ multiples of the speed of light $c$ per unit time $\Delta t$, where $N_{a}$ is any real number that is greater than or equal to one. Number $N_{a}$ will be referred to as the acceleration number.

$$
a_{x}=\left(\frac{N_{a} c}{\Delta t}\right) ; \quad N_{a} \in R ; N_{a} \geq 1
$$

Acceleration $a_{x}$ corresponds to the force $F_{x}$ per area $A_{0}$ acting on the cloud of accelerated charged particles, which correspond to pressure $P_{0}\left(F_{x}=A_{0} P_{0}\right)$ [2]. Area $A_{0}$ is also the cross sectional area traversed by 
the charged particles. The force $F_{x}$ can be expressed in terms of Newton's second law such that [2]:

$$
F_{x}=a_{x} m_{p}=\left(\frac{N_{a} c}{\Delta t}\right) m_{p}
$$

The momentum value $p_{x}$ is conventionally obtained by integrating force $F_{x}$ in respect to time $t$ with limits of integration from zero to time $\Delta t$ as shown below [2].

$$
p_{x}=\int_{a}^{\Delta t} F_{x} \mathrm{~d} t=N_{a} c m_{p}
$$

Keep in mind that momentum value $p_{x}$ corresponds to the relativistic energy value of $E=p_{x} c=N_{a} m_{p} c^{2}$; where the particles' mass value $m_{p}$ in energy $E$ is treated as rest mass due to the fact that the charged particles are massive particles and not relativistic particles such as photons [2]. Acceleration number $N_{a}$ varies proportionally to force $F_{x}$. Thus momentum $p_{x}$ corresponds to force $F_{x}$, however it is well understood that the charged particles of inertial mass $m_{p}$ cannot obtain super luminous velocities, therefore the particles' approximate momentum will be denoted $p_{a}$ as shown in Equation (1.03) below.

$$
p_{a}=v_{p}^{\prime} m_{p}^{\prime}
$$

As acceleration $a_{x}$ increases, the particles' velocity asymptotically approach but never achieve the speed of light; to circumvent this infinite decimal expansion, the asymptotic increase is set equal to the constant approximate of velocity denoted $v_{p}^{\prime}$. Velocity $v_{p}^{\prime}$ is the particles' approximate velocity "close to" but less than the speed of light as shown by Equation (1.04). Velocity $v_{p}^{\prime}$ is approximated to 99 percent of the speed of light for the purposes of this formulation.

$$
v_{p}^{\prime}=(0.99 \times c) ; v_{p}^{\prime}<c
$$

Mass value $m_{p}^{\prime}$ corresponding to momentum $p_{a}$ is referred to as variable inertial mass. Variable inertial mass $m_{p}^{\prime}$ takes on values greater than or equal to inertial mass $m_{p}$.

$$
m_{p}^{\prime} \geq m_{p}
$$

To avoid confusion, it must be noted that relativistic mass dilation is different from the variation of variable mass or inertial $m_{p}^{\prime}$ as the particle approaches the speed of light. This can be conveyed by setting variable inertial mass $m_{p}^{\prime}$ equal to the product of the variable inertial mass and the Lorentz factor $\gamma$ as shown below [2].

$$
m_{p}^{\prime}=m_{p}^{\prime} \gamma
$$

This can alternatively be expressed such that [2]:

$$
m_{p}^{\prime}=m_{p}^{\prime} \gamma=m_{p}^{\prime}\left[1-\frac{v^{2}}{c^{2}}\right]^{-1 / 2}
$$

Equation (1.06) implies that the Lorentz factor $\gamma$ is equal to 1 and implies that relative velocity $v$ or the velocity of an observer is zero $(v=0)$ for the purpose of this derivation.

$$
m_{p}^{\prime}=m_{p}^{\prime}\left[1-\frac{(0)^{2}}{c^{2}}\right]^{-1 / 2}
$$

Thus inertial mass $m_{p}^{\prime}$ does not vary according to the Lorentz factor $\gamma$. Conclusively, relativistic mass is dependent on the velocity and orientation of an observer while the inertial varies according to momentum. In continuing the formulation, momentum value $p_{a}$ is set equal to momentum $p_{x}$.

$$
p_{a}=p_{x}
$$

This equivalence can be expressed as:

$$
N_{a} c m_{p}=v_{p}^{\prime} m_{p}^{\prime}
$$

Equation (1.10) represents an important aspect of the assertion. The charged particles of the accelerated mass cannot exceed the speed of light, thus, variable inertial mass $m_{p}^{\prime}$ must vary proportionally to the acceleration 
number $N_{a}$; where the values $c, v_{p}^{\prime}$, and $m_{p}$ are constant. At this juncture, one solves for variable inertial mass $m_{p}^{\prime}$. Variable inertial mass $m_{p}^{\prime}$ is such that:

$$
m_{p}^{\prime}=\frac{N_{a} c m_{p}}{v_{p}^{\prime}}=\frac{N_{a} c m_{p}}{(0.99 \times c)} ; \quad m_{p}^{\prime} \geq m_{p}
$$

Equation (1.11) can be expressed such that:

$$
m_{p}^{\prime}=\frac{N_{a} m_{p}}{(0.99)}
$$

The premise of the assertion is the correlation of the acceleration of a cloud of charged particles and gravitation, therefore Newtonian gravitational force $F_{g}$ is given such that [2]:

$$
F_{g}=\frac{G M m}{r^{2}}
$$

The density of the accelerated cloud of charged particles is denoted $\rho_{p}$ where $N_{p}$ is the discrete number of charged particles in the cloud per unit volume $(V)$ as displayed below.

$$
\rho_{p}=\frac{m_{p}^{\prime} N_{p}}{V}
$$

This implies that:

$$
\rho_{p} V=m_{p}^{\prime} N_{p} \equiv M
$$

where $M$ is the source mass in gravitational force $F_{g}$. In the task of defining gravitation in terms of the varying inertial mass $m_{p}^{\prime}$ associated with the accelerated cloud of charged particles, again, consider Newtonian gravitational force $F_{g}[2]$.

$$
F_{g}=\frac{G M m}{\left|x_{\amalg}\right|^{2}}
$$

where $x_{\amalg}$ are the spatial coordinates in $R^{n} \quad\left(x_{\amalg} \in R^{n}\right)$, mass $M$ in Equation (1.16) (or Newtonian gravitational force) is substituted according to expression $1.15\left(m_{p}^{\prime} N_{p} \equiv M\right)$. Newtonian gravitational force can then be expressed such that:

$$
F_{g}=\frac{G m_{p}^{\prime} N_{p} m_{1}}{\left|x_{\text {u }}\right|^{2}}
$$

Mass value $m_{1}$ is the mass of the particle at distance $\left|x_{\mathrm{u}}\right|$ under the influence of the gravitational force produced by the cloud of accelerated charged particles. Substituting the value of inertial mass $m_{p}^{\prime}$ into Equation (1.17); gravitational force $F_{g}$ can then be expressed such that:

$$
F_{g}=G \frac{N_{p} N_{a} m_{p} m_{1}}{(0.99)\left(\left|x_{\text {щ }}\right|^{2}\right)}
$$

Therefore, the accelerating force $F_{x}$ acting on the charged particles is oriented in the direction of travel while the force of gravity $F_{g}$ generated by the particles is orthogonal to the direction of travel and therefore force $F_{x}$. Section 2 will introduce the correlation of the gravitational space-time curve associated with the gravitational field generated by the accelerated charged particles.

\section{Gravitation Produced by Accelerated Charged Particles and Einstein's Field Equations}

The gravitational field generated by the cloud of accelerated particles on the verge of the speed of light inherently produces a space-time curve. Therefore the mathematical description of the space-time curve produced by the accelerated charged particles is given by Einstein's field equation. Consider the function of expression (2.0) 
below.

$$
G_{\text {u }}\left(N_{p}, N_{a}\right)=\nabla_{\text {ц }} \phi\left(x_{\text {щ }}\right)
$$

The function $G_{\mathrm{u}}\left(N_{p}, N_{a}\right)$ is a surjective or "onto" map from the domain composed of the Cartesian product $N \times R$ to the codomain of n-dimensional real numbers $R^{n}$ [3]. Map $G_{\text {u }}$ has a domain of the number $N_{p}$ of charged particles in the density of the cloud of accelerated particles which is an element of natural numbers ( $\left.N_{p} \in N\right)$ and the acceleration number $N_{a}$ which is an element of real numbers $\left(N_{a} \in R\right)$. Map $G_{\mathrm{u}}$ is expressed as shown below [3].

$$
G_{\mathrm{u}}: N \times R \rightarrow R^{n}
$$

The symbol $\phi$ denotes the field function to which the space-time curve or manifold is defined [3]. In more technical terms, the field $\phi$ represents the differential manifold to which the metric tensor $g_{\mathrm{uv}}$ is defined on [4]. Thus function $\nabla_{\mathrm{u}} \boldsymbol{\phi}$ is a map from the set of Minkowski coordinates $x_{u}$ [4] in set $\boldsymbol{R}^{4}\left(x_{u} \in \boldsymbol{R}^{4}\right)$ to the codomain of the set of points and displacements in set $M^{n}\left(M^{n} \subset R^{n}\right)$ which is the set of points and displacements on the n-dimensional differential manifold or space-time curve surface. Where $\nabla_{\mathrm{u}}\left(\nabla_{\mathrm{u}}=\partial / \partial x_{\mathrm{u}}\right)$ is a partial derivative operator in respect to $x_{u}$, the function $\nabla_{\mathrm{u}} \phi$ is a map such that [3]:

$$
\nabla_{\mathrm{u}} \boldsymbol{\phi}: \boldsymbol{R}^{n} \rightarrow M^{n}
$$

The equivalence of function $G_{\mathrm{u}}\left(N_{p}, N_{a}\right)$ and function $\nabla_{\mathrm{u}} \phi\left(x_{\mathrm{u}}\right)$ imply a composition of functions $\left(\nabla_{\mathrm{L}} \phi\right) \circ G_{\mathrm{u}}$. Thus, Equation (2.0) expresses the composition map such that:

$$
\left(\nabla_{\mathrm{u}} \phi\right) \circ G_{\mathrm{u}}: N \times R \rightarrow M^{n}
$$

Thus, for every value $N_{p}$ and $N_{a}$ in the domain of map $\left(\nabla_{\mathrm{u}} \boldsymbol{\phi}\right) \circ G_{\mathrm{u}}$ there exist a value $a\left(\left(\nabla_{\mathrm{u}} \boldsymbol{\phi}\right) \circ G_{\mathrm{u}}=\boldsymbol{a}\right)$ such that $a$ is an element of set $M^{n}\left(a \in M^{n}\right)$, hence the "onto" or surjective mapping. Expression 2.0 is the correspondence of the number of particles $N_{p}$ and acceleration number $N_{a}$ to solutions of Einstein's field equation which are defined on a space-time manifold or set $M^{n}$. It will now be shown that the function of $G_{u}\left(N_{p}, N_{a}\right)=\nabla_{\mathrm{u}} \phi\left(x_{\mathrm{u}}\right)$ correspond to given solutions of the Einstein Field equations. Let function $\nabla_{\mathrm{u}} \phi\left(x_{\mathrm{u}}\right)$ equal a value such that:

$$
\nabla_{\mathrm{u}} \phi\left(x_{\mathrm{u}}\right)=A g_{\mathrm{uv}}+B
$$

where $A$ and $B$ are arbitrary values, $g_{\mathrm{uv}}$ is the metric tensor, and $A g_{\mathrm{uv}}+B$ is an element of the set of values on the n-dimensional differential manifold $M^{n}\left(\left(A g_{\mathrm{uv}}+B\right) \in M^{n}\right)$. The Einstein tensor $G_{\mathrm{uv}}$ is set equal function $\nabla_{ц} \phi\left(x_{\Perp}\right)$ such that [3]:

$$
G_{\mathrm{uv}}=\nabla_{\mathrm{u}} \phi\left(x_{\mathrm{u}}\right)=A g_{\mathrm{uv}}+B
$$

The Einstein tensor is given such that [3]:

$$
G_{\mathrm{uv}}=R_{\mathrm{uv}}-\frac{g_{\mathrm{uv}} R}{2}=g_{\mathrm{uv}}\left[\nabla_{s}, \nabla_{t}\right]-\frac{g_{\mathrm{uv}} R}{2}
$$

[where $R_{\mathrm{uv}}$ is the Ricci tensor, $R$ is the scalar constant, and $\left[\nabla_{s}, \nabla_{t}\right]$ is the differential commutator for computing curvature [3]. This implies that the values of $A$ and $B$ in $A g_{\mathrm{uv}}+B$ are such that:

$$
A=\left[\nabla_{s}, \nabla_{t}\right]-\frac{R}{2}, B=0
$$

As a second example, function $\nabla_{\mathrm{u}} \phi\left(x_{\mathrm{u}}\right)$ is now set equal to the stress-energy tensor $T_{\mathrm{uv}}$ for a perfect fluid as shown below.

$$
T_{\mathrm{uv}}=\nabla_{\mathrm{u}} \phi\left(x_{\mathrm{u}}\right)=A g_{\mathrm{uv}}+B
$$

The Stress-energy tensor is given such that [3]:

$$
T_{\mathrm{uv}}=\nabla_{\mathrm{u}} \phi \nabla_{v} \phi+g_{a b} \mathcal{L}_{\mathrm{KG}}=A g_{\mathrm{uv}}+B
$$

[where $\mathcal{L}_{K G}$ is the Lagrangian for the Klein-Gordon equation [3]] This implies that the values of $A$ and $B$ in 
$A g_{\mathrm{u} v}+B$ are such that:

$$
A=\mathcal{L}_{\mathrm{KG}}, \quad B=\nabla_{a} \phi \nabla_{\mathrm{L}} \phi
$$

Due to the equivalence of function $G_{\text {u }}\left(N_{p}, N_{a}\right)$ and function $\nabla_{\mathrm{u}} \phi\left(x_{\mathrm{u}}\right) \quad\left(G_{\mathrm{u}}\left(N_{p}, N_{a}\right)=\nabla_{\mathrm{u}} \phi\left(x_{\mathrm{u}}\right)\right)$ or $\left(\nabla_{\mathrm{u}} \phi\right) \circ G_{\mathrm{u}}$; this Implies that [3]:

$$
G_{\mathrm{u}}\left(N_{p}, N_{a}\right)=A g_{\mathrm{uv}}+B ; \forall A, B \in M^{n}
$$

Therefore the components of the Einstein tensor and the stress-energy tensor reside in the codomain of function $\left(\nabla_{\mathrm{u}} \phi\right) \circ G_{\mathrm{u}}$. This paper presents two derivations of Einstein's field equations that express the equivalence of the functions $G_{\mathrm{u}}\left(N_{p}, N_{a}\right)=\nabla_{\mathrm{u}} \phi\left(x_{\mathrm{u}}\right)$. Therefore, there are two descriptions of the space-time curve generated by the cloud of accelerated charged particles using two separate aspects of Einstein's field equations. The first description is the Schwarzchild aspect and the second is the stress-energy aspect as will be formulated in the next section.

\section{The Schwarzchild and Stress-Energy Description of a Space-Time Curve Generated by a Cloud of Accelerated Particles}

Section 3 will introduce two formulations linking the number of particles $N_{p}$ and acceleration number $N_{a}$ to solutions to Einstein's field equations, hence, validating the equivalence of $G_{\mathrm{u}}\left(N_{p}, N_{a}\right)=\nabla_{\mathrm{u}} \phi\left(x_{\mathrm{u}}\right)$. The formulation begins with the Schwarzchild description.

The Schwarzchild descritption

In reference to this hypothetical description, accelerated charged particles traveling at velocities bordering the speed of light generate a gravitational field on a spherically symmetric body, hence, the need to formulate a description using the Schwarzchild metric. The Schwarzchild radius is given such that [3]:

$$
r_{\mathrm{s}}=\left|x_{\mathrm{u}}\right|
$$

Gravitational force $F_{g}$ of Equation (1.18) is expressed in terms of the Schwarzchild radius $r_{s}$ as shown below.

$$
F_{g}=G \frac{N_{p} N_{a} m_{p} m_{1}}{(0.99)\left(r_{s}\right)^{2}}
$$

Gravitational potential energy $U_{g}$ is conventionally obtained by evaluating the integral in respect to Schwarzchild radius $r_{s}$ giving a value such that [3]:

$$
U_{g}=\int F_{g} \mathrm{~d} r_{s}=G \frac{N_{p} N_{a} m_{p} m_{1}}{(0.99)\left(r_{s}\right)}
$$

The maximum value of kinetic energy $K_{\max }$ for a particle of mass $m_{1}$ in the fluid with a maximum velocity at the speed of light $c$ is given such that [2]:

$$
K_{\max }=\frac{c^{2} m_{1}}{2}
$$

As is conventionally performed, kinetic energy $K_{\max }$ is set equal to gravitational potential energy $U_{g}$ as shown below [2].

$$
K_{\max }=U_{g}
$$

This equivalence can be expressed as [2]:

$$
\frac{c^{2} m_{1}}{2}=G \frac{N_{p} N_{a} m_{p} m_{1}}{(0.99)\left(r_{s}\right)}
$$

Solving for the Schwarzchild radius $r_{s}$ gives the radius in terms of the number of particles $N_{p}$ and acceleration number $N_{a}$ as shown below [2].

$$
r_{s}=G \frac{2 N_{p} N_{a} m_{p}}{(0.99)\left(c^{2}\right)}
$$


The Schwarzchild metric is given such that [3]:

$$
\mathrm{d}^{\prime} \tau^{2}=B^{\prime}(r) \mathrm{d} t^{2}-A^{\prime}(r) \mathrm{d} r^{2}-r^{2} \mathrm{~d} \theta^{2}-r^{2} \sin ^{2} \theta \mathrm{d} \Theta^{2}
$$

The functions $B^{\prime}(r)$ and $A^{\prime}(r)$ take on values of the Schwarzchild radius $r_{s}$ such that [3]:

$$
\begin{gathered}
B^{\prime}(r)=\left(1-\frac{r_{s}}{r}\right)=\left(1-G \frac{2 N_{p} N_{a} m_{p}}{(r)(0.99)\left(c^{2}\right)}\right) \\
A^{\prime}(r)=\left(1-\frac{r_{s}}{r}\right)^{-1}=\left(1-G \frac{2 N_{p} N_{a} m_{p}}{(r)(0.99)\left(c^{2}\right)}\right)^{-1}
\end{gathered}
$$

[where $r$ is the radius of the spherically symmetric body $r=(A / 4 \pi)^{1 / 2}$ [3]] The prime notation (') denotes that the variations in the metric correspond to the number of particles $N_{p}$ and acceleration number $N_{a}$. The metric $\mathrm{d}^{\prime} \tau^{2}$ is set equal to the metric tensor $g_{\mathrm{Lv}}^{\prime}$.

$$
\mathrm{d}^{\prime} \tau^{2}=g_{\mathrm{uv}}^{\prime}
$$

Expressing the value of metric tensor $g_{\mathrm{uv}}^{\prime}$ gives the matrix expression of:

$$
g_{\mathrm{uv}}^{\prime}=\left[\begin{array}{cccc}
\left(1-G \frac{2 N_{p} N_{a} m_{p}}{(r)(0.99)\left(c^{2}\right)}\right) & 0 & 0 \\
0 & -\left(1-G \frac{2 N_{p} N_{a} m_{p}}{(r)(0.99)\left(c^{2}\right)}\right)^{-1} & 0 & 0 \\
0 & -r^{2} & 0 \\
0 & 0 & 0 & -r^{2} \sin ^{2} \theta
\end{array}\right]
$$

Therefore, the Einstein tensor $G_{\mathrm{uv}}^{\prime}$ can be expressed such that [3]:

$$
G_{\mathrm{uv}}^{\prime}=R_{\mathrm{uv}}^{\prime}-\frac{g_{\mathrm{uv}}^{\prime} R}{2} \equiv 0
$$

where the Ricci tensor $R_{\mathrm{uv}}^{\prime}$ is expressed in terms of the differential commutator for computing curvature $\left[\nabla_{s}, \nabla_{t}\right]$ such that $[3]$ :

$$
R_{\mathrm{uv}}^{\prime}=g_{\mathrm{uv}}^{\prime}\left[\nabla_{s}, \nabla_{t}\right]
$$

The requirement of the equivalence of $G_{\mathrm{u}}\left(N_{p}, N_{a}\right)=\nabla_{\mathrm{u}} \phi\left(x_{\mathrm{u}}\right) \quad\left(G_{\mathrm{u}}: N \times R \rightarrow M^{n}\right)$ is satisfied where function $\nabla_{\mathrm{u}} \phi\left(x_{\mathrm{u}}\right)$ is substituted by function $\nabla_{\mathrm{u}} \phi(\boldsymbol{t}, \boldsymbol{r}, \theta, \Theta)$ at time $t$, spherical radius $r$, and spherical angles $\theta$ and $\Theta$ indicating the spherical coordinates of the Schwarzchild metric.

$$
G_{\mathrm{u}}\left(N_{p}, N_{a}\right)=\nabla_{\mathrm{u}} \phi\left(x_{\mathrm{u}}\right) \equiv \nabla_{\mathrm{u}} \phi(\boldsymbol{t}, \boldsymbol{r}, \theta, \Theta)
$$

The stress-energy tensor description

A cloud of charged particles are again accelerated via an electromagnetic force (of any given source i.e. particle accelerator or subatomic charged particles emitted from a star) to the verge of the speed of light producing a gravitational field that is exerting on a fluid of particles of mass $m_{1}$ per unit volume. For the purpose of this derivation, the fluid is considered a perfect fluid. Thus, the stress energy tensor for a perfect fluid $T_{a b}$ is expressed as shown below [3].

$$
T_{a b}=u_{a} u_{b}+g_{a b} \mathcal{L}_{K G}
$$

where $\mathcal{L}_{\mathrm{KG}}$ is the Lagrangian for the Klein-Gordon equation as shown below [3].

$$
\mathcal{L}_{K G}=\frac{1}{2}\left[\nabla_{c} \phi \nabla^{c} \phi-\phi^{2} m_{1}^{2}\right]
$$


The fluid 4-velocity denoted $u_{\text {и }}$ varies along a geodesic embedded on the space-time manifold. Fluid velocity $u_{\text {и }}$ progresses along a time-like curve and can be expressed in terms of the chain rule [5] as expressed below [3].

$$
u_{\mathrm{u}}=\frac{\partial \phi}{\partial x_{\mathrm{u}}} \frac{\partial x_{\mathrm{u}}}{\partial t}=\frac{\partial \phi}{\partial t}=\nabla_{\text {щ }}
$$

The geodesic rule is acknowledged as shown below [3].

$$
\nabla_{a}\left(\nabla_{ц} \phi\right)=\mathbf{0}
$$

Hence the appropriate use of the Christoffel symbol $\Gamma$ applies as follows [3].

$$
\nabla_{a}\left(\nabla_{\mathrm{u}} \boldsymbol{\phi}\right)=\nabla_{a}\left(\nabla_{\mathrm{u}} \boldsymbol{\phi}\right)+\Gamma\left(\nabla_{\mathrm{u}} \boldsymbol{\phi}\right) \equiv \mathbf{0}
$$

In substituting fluid velocity $u_{u}$ for the differential notation of $\nabla_{u} \phi$ in Equation (3.15), the stress-energy tensor is expressed such that [3]:

$$
T_{a b}=\nabla_{a} \phi \nabla_{b} \phi+g_{a b} \mathcal{L}_{K G}
$$

Consider dynamic pressure $P_{d}$ at fluid velocity $u_{\text {и }}$ and fluid density $\rho$ shown below.

$$
P_{d}=\frac{\rho}{2} \sum_{i=0}^{3} u_{u}^{2}
$$

At this juncture, fluid velocity $u_{\mathrm{u}}$ is substituted with the time-like partial derivative $\nabla_{\mathrm{u}} \phi$ in dynamic pressure $P_{d}$ as shown below [2].

$$
P_{d}=\frac{\rho}{2} \sum_{i=0}^{3} u_{\mathrm{u}}^{2}=\frac{\rho}{2} u_{a} \cdot u_{b}=\frac{\rho}{2} \nabla_{a} \phi \nabla_{b} \phi
$$

Or alternatively,

$$
P_{d}=\frac{\rho}{2} \nabla_{a} \phi \nabla_{b} \phi
$$

Let dynamic pressure $P_{d}$ equal the expression of the sums of components of partial pressure $P_{\mathrm{u}}$, where each component of pressure $P_{d}$ is a part of the diagonal components of the 4 by 4 matrix of stress-energy tensor $T_{a b}\left(P_{\mathrm{u}}+g_{a b} \mathcal{L}_{\mathrm{KG}}=\operatorname{diag}\left[T_{a b}\right]\right)$.

$$
P_{d}=\sum_{i=0}^{3} P_{\Perp}
$$

This implies that:

$$
\sum_{i=0}^{3} P_{\text {щ }}=\frac{\rho}{2} \nabla_{a} \phi \nabla_{b} \phi
$$

Isolating the partial derivatives $\nabla_{a} \phi \nabla_{b} \phi$, gives:

$$
\nabla_{a} \phi \nabla_{b} \phi=\frac{2}{\rho} \sum_{i=0}^{3} P_{\text {แ }}
$$

Substituting Equation (3.26) into Equation (3.20) (or the stress tensor), one obtains:

$$
T_{a b}=\frac{2}{\rho} \sum_{i=0}^{3} P_{\text {щ }}+g_{a b} \mathcal{L}_{\text {KG }}
$$

Consider the unit vector $u$ in $R^{4}$ shown below [5].

$$
u=\frac{x_{\mathrm{u}}}{\left|x_{\mathrm{u}}\right|}
$$

Gravitational force $F_{g}$ generated by accelerated particles is multiplied by unit vector $u$ giving a vector valued force $\left(F_{g}\right)_{\text {ц }}[5]$. 


$$
\left(F_{g}\right)_{\text {ч }}=F_{g} u=G \frac{x_{u} N_{p} N_{a} m_{p} m_{1}}{(0.99)\left(\left|x_{u}\right|^{3}\right)}
$$

Using the classical equation of pressure equal to force per unit area $(P=F / A)$ [2], components of pressure $P_{\text {ц }}$ is set equal to the components of the ratio of vector valued gravitational force $\left(F_{g}\right)_{\text {щ }}$ and spherically symmetric area $A^{\prime} \quad\left(A^{\prime}=n \pi\right)$ perpendicular to force as shown below.

$$
P_{\text {щ }}=\frac{\left(F_{g}\right)_{\text {щ }}}{A^{\prime}}=G \frac{x_{u} N_{p} N_{a} m_{p} m_{1}}{A^{\prime}(0.99)\left(\left|x_{\text {u }}\right|^{3}\right)}
$$

The sums of components of pressure $P_{\text {ч }}$ are equal to the sums of components $\left(F_{g}\right)_{\text {ч }}$ such that:

$$
\sum_{i=0}^{3} P_{\text {щ }}=\sum_{i=0}^{3} G \frac{x_{\text {щ }} N_{p} N_{a} m_{p} m_{1}}{A^{\prime}(0.99)\left(\left|x_{\text {щ }}\right|^{3}\right)}
$$

Substituting the value of Equation (3.31) into Equation (3.27) gives the stress energy tensor $T_{a b}$ such that:

$$
T_{a b}=\frac{2}{\rho} \sum_{i=0}^{3} G \frac{x_{\mathrm{u}} N_{p} N_{a} m_{p} m_{1}}{A^{\prime}(0.99)\left(\left|x_{\mathrm{u}}\right|^{3}\right)}+g_{a b} \mathcal{L}_{K G}
$$

The stress energy tensor is set equal to the Einstein tensor $G_{a b}\left(T_{a b}=G_{a b}\right)$ to show the correspondence between the gravitational stress $T(T=F / A)$ exerted on a perfect fluid which flows through a region of space-time and the curved geometry of that region of space-time to which the fluid travels. Thus, Equation (3.32) below gives a full description of the gravitational effects of the cloud of accelerated charged particles on both the dynamics of the fluid and the curved surface of the space-time maniflold.

$$
\frac{2}{\rho} \sum_{i=0}^{3} G \frac{x_{\mathrm{u}} N_{p} N_{a} m_{p} m_{1}}{A^{\prime}(0.99)\left(\left|x_{\mathrm{u}}\right|^{3}\right)}+g_{a b} \mathcal{L}_{K G}=R_{a b}-\frac{g_{a b} R}{2} \equiv 0
$$

Conclusively, the stress-energy tensor describing the pressure exerted by the gravitational field produced by the accelerated charged particles on a perfect fluid correspond to the surjective map of $G_{\mathrm{u}}\left(N_{p}, N_{a}\right)=\nabla_{\mathrm{u}} \phi\left(x_{\Perp}\right)$ (or $\left(\nabla_{\mathrm{u}} \phi\right) \circ G_{\mathrm{u}}: N \times R \rightarrow M^{n}$ ). Therefore it has been effectively shown to correlate the variations in the number of particles $N_{p}$ and acceleration number $N_{a}$ to solutions to Einstein's field equation.

\section{The Generation of Electromagnetic Force to Exert Sufficient Acceleration to Produce a Gravitational Field}

It is of great importance to show the possibility and feasibility of accelerating a cloud of charged particles to an extent to where they actually produce a gravitational field in the real world. Thus, the Lorentz equation of electromagnetic force is applied to show this possibility. Lorentz force $F_{L}$ is as shown below [2].

$$
F_{L}=q[\bar{E}+\bar{v} \times \bar{B}]
$$

The velocity vector $\bar{v}$ is the velocity of each individual charged particle in the cloud density being accelerated by vector valued electromagnetic force $F_{L}$ [2]. Where $q$ is the individual charge of each particle in the cloud density. The x-component of particle velocity $\bar{v}$ is given as approximated velocity value $v_{p}^{\prime}$ as shown below.

$$
\bar{v}=\left(v_{p}^{\prime}, 0,0\right)=((0.99 \times c), 0,0)
$$

The vector value for the magnetic field is given such that:

$$
\bar{B}=(0, B, 0)
$$

The vector value for the electric field is given such that:

$$
\bar{E}=(E, 0,0)
$$


Carrying out the cross product of velocity vector $\bar{v}$ and magnetic field vector $\bar{B}$ give the orthogonal vector value shown below.

$$
\bar{v} \times \bar{B}=\left(0,0, v_{p}^{\prime} B\right)
$$

The value of Lorentz force vector $F_{L}$ at the given vector quantities of electric field $\bar{E}$, particle velocity $\bar{v}$, and magnetic field $\bar{B}$ are that of Equation (4.05) below.

$$
F_{L}=q[\bar{E}+\bar{v} \times \bar{B}]=\left(q E, 0, q v_{p}^{\prime} B\right)
$$

The magnitude of electromagnetic force vector $F_{L}\left(\left|F_{L}\right|\right)$ takes on a value such that:

$$
\left|F_{L}\right|=\left[(q E)^{2}+\left(q v_{p}^{\prime} B\right)^{2}\right]^{1 / 2}
$$

The magnitude of electromagnetic force $\left|F_{L}\right|$ is set equal to force $F_{x}$ corresponding to pressure $P_{0}$ and cross sectional area $A_{0}$ (previously mentioned in section 1 ) to be acting on the cloud of charged particles as shown in Equation (4.07) below.

$$
\left|F_{L}\right|=F_{x}=A_{0} P_{0}
$$

Recall that acceleration $a_{x}$ corresponds to the force $\left(F_{x}\right)$ per unit area $\left(A_{0}\right)$ acting on the cloud of accelerated charged particles, which correspond to pressure $P_{0}$ (where $F_{x}=A_{0} P_{0}$ ). Recall that force $F_{x}$ takes on a value such that:

$$
F_{x}=a_{x} m_{p}=\left(\frac{N_{a} c}{\Delta t}\right) m_{p}
$$

The value of Equation (4.07) then becomes:

$$
\left|F_{L}\right|=\left(\frac{N_{a} c}{\Delta t}\right) m_{p}
$$

Equation (4.09) can be expressed such that:

$$
\left(\frac{N_{a} c}{\Delta t}\right) m_{p}=q\left[(E)^{2}+\left(v_{p}^{\prime} B\right)^{2}\right]^{1 / 2}
$$

The task is to obtain the required voltage at a given acceleration number $N_{a}$, this will require one to solve Equation (4.10) for electric field $E$ as shown below.

$$
E=\left[\left(\frac{m_{p} N_{a} c}{q \Delta t}\right)^{2}-\left(v_{p}^{\prime} B\right)^{2}\right]^{1 / 2}
$$

Recall that velocity $v_{p}^{\prime}$ is the particles' approximate velocity at $99 \%$ of the speed of light. Thus, velocity $v_{p}^{\prime}$ is simply the product of the speed of light $c$ and the value $0.99\left(v_{p}^{\prime}=(0.99 \times c)\right)$. The speed of light $c$ can then be distributed out of Equation (4.11), giving the value of Equation (4.12) such that:

$$
E=c\left[\left(\frac{m_{p} N_{a}}{q \Delta t}\right)^{2}-((0.99) B)^{2}\right]^{1 / 2}
$$

The value of electrical field $E$ is equal to the negative partial derivative of voltage $V$ in respect to length $x$ [2].

$$
E=-\frac{\partial V}{\partial x}
$$

Substituting this value (Equation (4.13)) into Equation (4.12) gives the differential equation shown below.

$$
\frac{\partial V}{\partial x}=-C\left[\left(\frac{m_{p} N_{a}}{q \Delta t}\right)^{2}-((0.99) B)^{2}\right]^{1 / 2}
$$


This can be rearranged such that:

$$
\partial V=\left(-c\left[\left(\frac{m_{p} N_{a}}{q \Delta t}\right)^{2}-((0.99) B)^{2}\right]^{\frac{1}{2}}\right) \partial x
$$

The corresponding integrals in respect to voltage $V$ and length $x$ are expressed such that [2]:

$$
\left.\int_{V_{i}=0}^{V_{f}=V_{a}} \partial V=\int_{x_{i}=0}^{x_{f}=\zeta}-C\left[\left(\frac{m_{p} N_{a}}{q \Delta t}\right)^{2}-((0.99) B)^{2}\right]^{\frac{1}{2}}\right) \partial x
$$

where $\zeta$ is unit length, evaluating the integrals give the value of voltage $V_{a}$ such that:

$$
V_{a}=-c \zeta\left[\left(\frac{m_{p} N_{a}}{q \Delta t}\right)^{2}-((0.99) B)^{2}\right]^{\frac{1}{2}}
$$

Length $\zeta$ is set to unity $(\zeta=1)$, therefore voltage $V_{a}$ can be expressed such that:

$$
V_{a}=-c\left[\left(\frac{m_{p} N_{a}}{q \Delta t}\right)^{2}-((0.99) B)^{2}\right]^{\frac{1}{2}}
$$

Voltage $V_{a}$ is the product of electrical current $I$ and resistance $R\left(V_{a}=I R\right)$ [2].

$$
I R=-c\left[\left(\frac{m_{p} N_{a}}{q \Delta t}\right)^{2}-((0.99) B)^{2}\right]^{\frac{1}{2}}
$$

Equations ((4.18) and (4.19)) show the required voltage $V_{a}$ at acceleration number $N_{a}$ to produce gravitational force fields and the corresponding space-time curves using a given density number $N_{p}$ of charged particles at $99 \%$ if the speed of light. Thus the value of voltage $V_{a}$ or $I R$ sufficient to produce an acceleration that will generate gravity can be shown to exist in the real world with the condition of the inequality below.

$$
\left(\frac{m_{p} N_{a}}{q \Delta t}\right)^{2}>((0.99) B)^{2}
$$

Voltage $V_{a}$ can be mapped to and corresponds to a gravitational force value $F_{g}$ at the number of particles $N_{p}$ and acceleration number $N_{a}$ as shown below.

$$
V_{a} \rightarrow F_{g}\left(N_{p}, N_{a}\right)
$$

where gravitational force $F_{g}\left(N_{p}, N_{a}\right)$ is such that:

$$
F_{g}\left(N_{p}, N_{a}\right)=G \frac{N_{p} N_{a} m_{p} m_{1}}{(0.99)\left(\left|x_{\mathrm{u}}\right|^{2}\right)}
$$

\section{Conclusion: Experiment Proposal}

The force associated with the Casimir effect describing vacuum energy was confirmed by an experiment conducted by physicist Steven Lamoreaux in 1996 [6]. The experiment was conducted in a vacuum between two metal plates [6]. The minutest value of force pulling the plates together was detected. Thus, in a similar manner, an experiment can be conducted where a cloud of charged particles are accelerated to within an infinitesimal fraction of the speed of light between two non-metal plates constructed of an electrically neutral material to avoid the detection of electromagnetic forces that can be confused with gravitational force. The equation of gra- 
vitational force $F_{g}$ linking gravitation to acceleration number $N_{a}$ and the number of particles $N_{p}$ can be used to predict a pressure ( $P=F_{g} / A_{p}$ ) exerted on the electrically neutral plates. The experimental verification of the equations formulated in this paper will lead to further progress in generating gravitational fields and space-time curves based on any method of energy generation that produces electromagnetic energy used to sufficiently accelerate the charged particles. Lastly, the obvious implication to the generation of a gravitational field proportionally to energy produced is the possibility of generating artificial gravity without the use of centripetal force and the generation of space-time curves also science fictionally referred to as warp fields.

\section{References}

[1] Manor, E. (2016) Journal of Modern Physics, 7, 422-425. http://dx.doi.org/10.4236/jmp.2016.75043

[2] Young, H.D. and Roger, F.A. (2004) Sears and Zemansky’s University Physics. 11th Edition, Cal., Pearson, Addison Wesley, San Francisco, 128-129, 417,441, 445, 516-517, 530, 893, 1024, 1410, 1431.

[3] Wald, R.M. (1984) General Relativity. Chicago Press, Ltd., Chicago, 23-26, 31-38, 70, 120-124.

[4] Penrose, R. (2004) The Road to Reality: Comprehensive Guide to the Laws of the Universe. Alfred A. Knopf Publishing, New York, 222-223.

[5] Stewart, J. (2003) Calculus. 5th Edition, Thompson, Brooks/Cole, Belmont, 968, 1095.

[6] http://math.ucr.edu/home/baez/physics/Quantum/casimir.html 